\title{
Search for OB stars running away from young star clusters
}

\section{NGC 6611}

\author{
V. V. Gvaramadze $\mathrm{e}^{1,2}$ and D. J. Bomans ${ }^{1}$ \\ 1 Astronomical Institute, Ruhr-University Bochum, Universitätstrasse 150, 44780 Bochum, Germany \\ e-mail: bomans@astro.rub.de \\ 2 Sternberg Astronomical Institute, Moscow State University, Universitetskij Pr. 13, Moscow 119992, Russia \\ e-mail: vgvaram@mx.iki.rssi.ru
}

Received 17 June 2008/ Accepted 2 September 2008

ABSTRACT

\begin{abstract}
$N$-body simulations have shown that the dynamical decay of the young ( $1 \mathrm{Myr}$ ) Orion Nebula cluster could be responsible for the loss of at least half of its initial content of OB stars. This result suggests that other young stellar systems could also lose a significant fraction of their massive stars at the very beginning of their evolution. To confirm this expectation, we used the Mid-Infrared Galactic Plane Survey (completed by the Midcourse Space Experiment satellite) to search for bow shocks around a number of young ( $\lesssim$ several Myr) clusters and OB associations. We discovered dozens of bow shocks generated by OB stars running away from these stellar systems, supporting the idea of significant dynamical loss of OB stars. In this paper, we report the discovery of three bow shocks produced by O-type stars ejected from the open cluster NGC 6611 (M16). One of the bow shocks is associated with the O9.5Iab star HD165319, which was suggested to be one of "the best examples for isolated Galactic high-mass star formation" (de Wit et al. 2005 , A\&A, 437, 247). Possible implications of our results for the origin of field OB stars are discussed.
\end{abstract}

Key words. stars: individual: BD-14 5040 - stars: individual: HD 165319 - open clusters and associations: general open clusters and associations: individual: NGC 6611 - ISM: individual objects: RCW 158

\section{Introduction}

Dynamical three- and four-body encounters between stars in the dense cores of young star clusters impart to some stars velocities exceeding the escape velocity from the cluster's potential well (Poveda et al. 1967; Leonard \& Duncan 1990). The concentration of massive stars towards the center of young clusters (e.g. Hillenbrand \& Hartmann 1998; Gouliermis et al. 2004; Chen et al. 2007) implies that the dynamical evolution of cluster cores is dominated by massive stars and that the majority of escaping stars should be massive. The escaping massive stars constitute a population of OB field stars (Gies 1987). The escape velocity ranges from several $\mathrm{km} \mathrm{s}^{-1}$ (for loose and low-mass clusters) to several tens of $\mathrm{km} \mathrm{s}^{-1}$ (for compact and massive ones), so that the typical space velocity of OB field stars is $\sim 10 \mathrm{~km} \mathrm{~s}^{-1}$ (e.g. Gies 1987). The stars with peculiar (transverse) velocities exceeding $30 \mathrm{~km} \mathrm{~s}^{-1}$ are called runaway stars (Blaauw 1961). It should be realized, however, that any star unbound from the parent cluster should be considered as a "runaway", independently of its peculiar velocity (cf. de Wit et al. 2005). Although the peculiar velocity of some runaway $\mathrm{OB}$ stars could be as high as several $100 \mathrm{~km} \mathrm{~s}^{-1}$ (e.g. Ramspeck et al. 2001) or even $\sim 500-700 \mathrm{~km} \mathrm{~s}^{-1}$ (Edelmann et al. 2005; Heber et al. 2008), the majority have velocities of several tens of $\mathrm{km} \mathrm{s}^{-1}$. Therefore, most runaway OB field stars should be located not far from their birth clusters.

A (massive) star moving supersonically with respect to the ambient medium generates a bow shock (Baranov et al. 1971; Van Buren \& McCray 1988) visible in infrared (Van Buren et al. 1995; Noriega-Crespo et al. 1997; France et al. 2007) and/or $\mathrm{H}_{\alpha}$ (Kaper et al. 1997; Brown \& Bomans 2005).
Observations of runaway OB stars showed that only a small fraction ( $\$ 20$ per cent) produce bow shocks (Van Buren et al. 1995; Noriega-Crespo et al. 1997; Huthoff \& Kaper 2002). The main reason for this is that many runaway stars move through the hot interstellar gas and their peculiar velocities are lower than the sound speed in the ambient medium (Huthoff \& Kaper 2002). The geometry of a bow shock allows us to infer the direction of motion of the associated star and thereby trace its trajectory backwards to the parent cluster in those cases in which the proper motion of the star has not been measured directly.

$N$-body simulations by Pflamm-Altenburg \& Kroupa (2006) showed that the dynamical decay of the young ( $\sim 1 \mathrm{Myr})$ Orion Nebula cluster could be responsible for the loss of at least half of its initial content of OB stars (see also Kroupa 2004; cf. Aarseth \& Hills 1972). This result suggests that other young stellar systems could also lose a significant fraction of their massive stars at the very beginning of their evolution. We therefore expect to observe numerous signatures of interaction between ejected stars and the dense environment of young star clusters. To confirm this expectation, we searched for bow shocks around a number of young ( several Myr) clusters and OB associations using the Mid-Infrared Galactic Plane Survey. This survey, carried out with the Spatial Infrared Imaging Telescope onboard the Midcourse Space Experiment (MSX) satellite (Price et al. 2001), covers the entire Galactic plane within $|b|< \pm 5^{\circ}$ and provides images at $18^{\prime \prime}$ resolution in four mid-infrared spectral bands centered at $8.3 \mu \mathrm{m}$ (band A), $12.1 \mu \mathrm{m}$ (band C), $14.7 \mu \mathrm{m}$ (band D), and $21.3 \mu \mathrm{m}$ (band E). To search for possible optical counterparts to the detected bow shocks, we used the SuperCOSMOS H-alpha Survey (SHS, Parker et al. 2005) and the Digitized Sky Survey (McLean et al. 2000). Our choice of young stellar 
systems implies that the majority of ejected stars should not have traveled far from their birth places (so that the parent clusters could be identified with more confidence) and that supernova explosions in massive binaries do not significantly contribute to the production of runaway stars (Blaauw 1961).

We discovered dozens of bow shocks. Three are discussed in this paper, while the results of study of other objects are presented elsewhere. The geometry of the three bow shocks suggests that they are driven by stars expelled from the young open star cluster NGC 6611. In Sect.2, we review the relevant data for this cluster. The results of the search for bow shocks around NGC 6611 are presented in Sect. 3 and discussed in Sect. 4. Conclusions are summarized in Sect. 5.

\section{NGC 6611}

NGC 6611 is part of the Ser OB 1 association in the Sagittarius spiral arm. It is embedded in the molecular cloud W37 and is responsible for ionizing the Eagle Nebula (M 16), famous for its "elephant trunks" and "EGGs" (evaporating gaseous globules, Hester et al. 1996). Numerous distance estimates for NGC 6611 converge to a figure $d \sim 1.75-2.00 \mathrm{kpc}$ (Hillenbrand et al. 1993; Loktin \& Beshenov 2003; Guarcello et al. 2007; Wolff et al. 2007). The age of the cluster is less well constrained. The presence of four early O-type stars in the cluster (e.g. Dufton et al. 2006) implies that its age is $\$ 2-3 \mathrm{Myr}$, while the association with the cluster of a B2.5I star (BD-13 4912) suggests that star formation in this part of Ser OB 1 started $~ 6 \mathrm{Myr}$ ago (Hillenbrand et al. 1993; de Winter et al. 1997; Kharchenko et al. 2005).

There is a growing body of evidence, however, that clustered star formation is a rapid process, so the age spread in the cluster should not exceed 1 Myr (e.g. Elmegreen 2000; BallesterosParedes \& Hartmann 2007). The evolutionary status of the majority of OB stars in NGC 6611 is consistent with an age of $\sim 4 \mathrm{Myr}$ and in the following we adopt this figure to be the age of the cluster. The observed age spread could be understood if the most massive (and therefore the youngest) stars in the cluster are the blue stragglers (the rejuvenated stars formed via merging of the less massive stars in the course of binary-single and binary-binary encounters; e.g. Leonard 1995; Portegies Zwart et al. 1999; Gvaramadze \& Bomans 2008), while the older stars are the former field stars trapped in the potential well of the contracting pre-cluster cloud (Pflamm-Altenburg \& Kroupa 2007). Numerous young $(\sim 0.1-3 \mathrm{Myr})$ pre-main-sequence stars surrounding NGC 6611 (e.g. Guarcello et al. 2007) could represent the second generation of stars, whose formation was triggered inside the remainder of the natal molecular cloud by the activity of massive stars in the cluster.

Radial-velocity measurements for OB stars in NGC 6611 indicated that $\simeq 30-50$ per cent are spectroscopic binaries (Bosch et al. 1999). The high percentage of massive binaries is one of the necessary conditions for production (e.g. via binary-binary encounters, Mikkola 1983; Leonard \& Duncan 1990) of runaway OB stars and merged (rejuvenated) massive stars (either bound or unbound to the cluster, Leonard 1995). Two other necessary conditions are the presence in the cluster of a sufficiently high number of massive binaries (i.e. the cluster should be massive) and the initially compact structure of the cluster. It is likely that both conditions were fulfilled in NGC 6611, at least at the beginning of its dynamical evolution.

A mass estimate for NGC 6611 suggests that the cluster could be as massive as $\simeq 2 \times 10^{4} M_{\odot}$ (Wolff et al. 2007). This estimate was derived by assuming that $\sim 160$ cluster members with masses ranging from 6 to $12 M_{\odot}$ constitute $\sim 5$ per cent of the total cluster mass (for a Salpeter initial mass function). Adopting this mass estimate, we infer that the cluster should contain $\sim 100$ OB stars, which exceeds the number of observed stars by a factor of 2.5. It is possible that the Salpeter initial mass function is not applicable for NGC 6611. Another possibility is that the cluster has already lost most of its massive stars by dynamical ejection (see also Sect. 4).

It is likely that NGC 6611 was initially more centrally concentrated and that its current size (the radius of the cluster is $\simeq 7-8$ pc for $d=2 \mathrm{kpc}$, Belikov et al. 2000; Bonato et al. 2006) is the result of dynamical decay of the cluster's core and overall cluster expansion caused by gas expulsion due to supernova explosions and stellar winds (e.g. Boily \& Kroupa 2003). The initially compact configuration would be consistent with the observation that the characteristic radius of young clusters is $\lesssim 1 \mathrm{pc}$, which is independent of their mass (Kroupa \& Boily 2002). For this radius and the above mass estimate, the escape velocity from the cluster is inferred to have been $\sim 10 \mathrm{~km} \mathrm{~s}^{-1}$.

Proper-motion measurements for NGC 6611 (see Table 1) suggest that the cluster is moving in the west-east direction with a peculiar (tangential) velocity of $\simeq 10-15 \mathrm{~km} \mathrm{~s}^{-1}$. To convert the observed proper motion into the true tangential velocity of the cluster, we used the Galactic constants $R_{0}=8 \mathrm{kpc}$ and $\Theta_{0}=200 \mathrm{~km} \mathrm{~s}^{-1}$ (e.g. Reid 1993; Kalirai et al. 2004; Avedisova 2005), the solar peculiar motion $\left(U_{\odot}, V_{\odot}, W_{\odot}\right)=$ $(10.00,5.25,7.17) \mathrm{kms}^{-1}$ (Dehnen \& Binney 1998), and adopted a distance of $d=2 \mathrm{kpc}$.

\section{Results of the search}

\subsection{Bow shocks}

We searched for bow shocks around NGC 6611 in a $12^{\circ}$ wide area elongated along the Galactic plane and centered on the cluster's longitude $\left(l=16^{\circ} .95\right)$. Along the Galactic latitude, the search was limited by the MSX coverage (see Sect. 1). At a distance of $1.75-2.00 \mathrm{kpc}, 1^{\circ}$ corresponds to $\simeq 30-34 \mathrm{pc}$, so that potentially we were able to detect bow shocks produced by stars leaving the cluster at the very beginning of its evolution (i.e. $\sim 4$ Myr ago) with a peculiar (tangential) velocity of $\$ 25-50 \mathrm{~km} \mathrm{~s}^{-1}$. We realize that some runaway OB stars were ejected at higher velocities and therefore could be beyond the area covered by our search. On the other hand, the lower the peculiar velocity, the lower the percentage of stars moving supersonically through the ambient medium. Thus, we expect that only a small fraction of runaway stars located within several degrees from the cluster are able to generate the bow shocks (see also Sect. 4)

The visual inspection of MSX images revealed several bowshock candidates. Three of them (indicated in Fig. 1 by circles) have a clear arc-like structure that opens towards NGC 6611 (Fig. 2-4), which suggests that these structures are generated by stars expelled from the cluster. The bow shocks 1 and 2 are most prominent at $21.3 \mu \mathrm{m}$ (Figs. 2 and 3), while the bow shock 3 is visible only at $8.3 \mu \mathrm{m}$ and $21.3 \mu \mathrm{m}$ (more clearly at $8.3 \mu \mathrm{m}$; Fig. 4). The bow shocks 2 and 3 have obvious optical counterparts in the SHS (see Figs. 3 and 4), which are the parts of H II regions, respectively, G 016.9-01.1 (Kuchar \& Clark 1997) and RCW 158 (Rodgers et al. 1960; see also Sect. 4). 


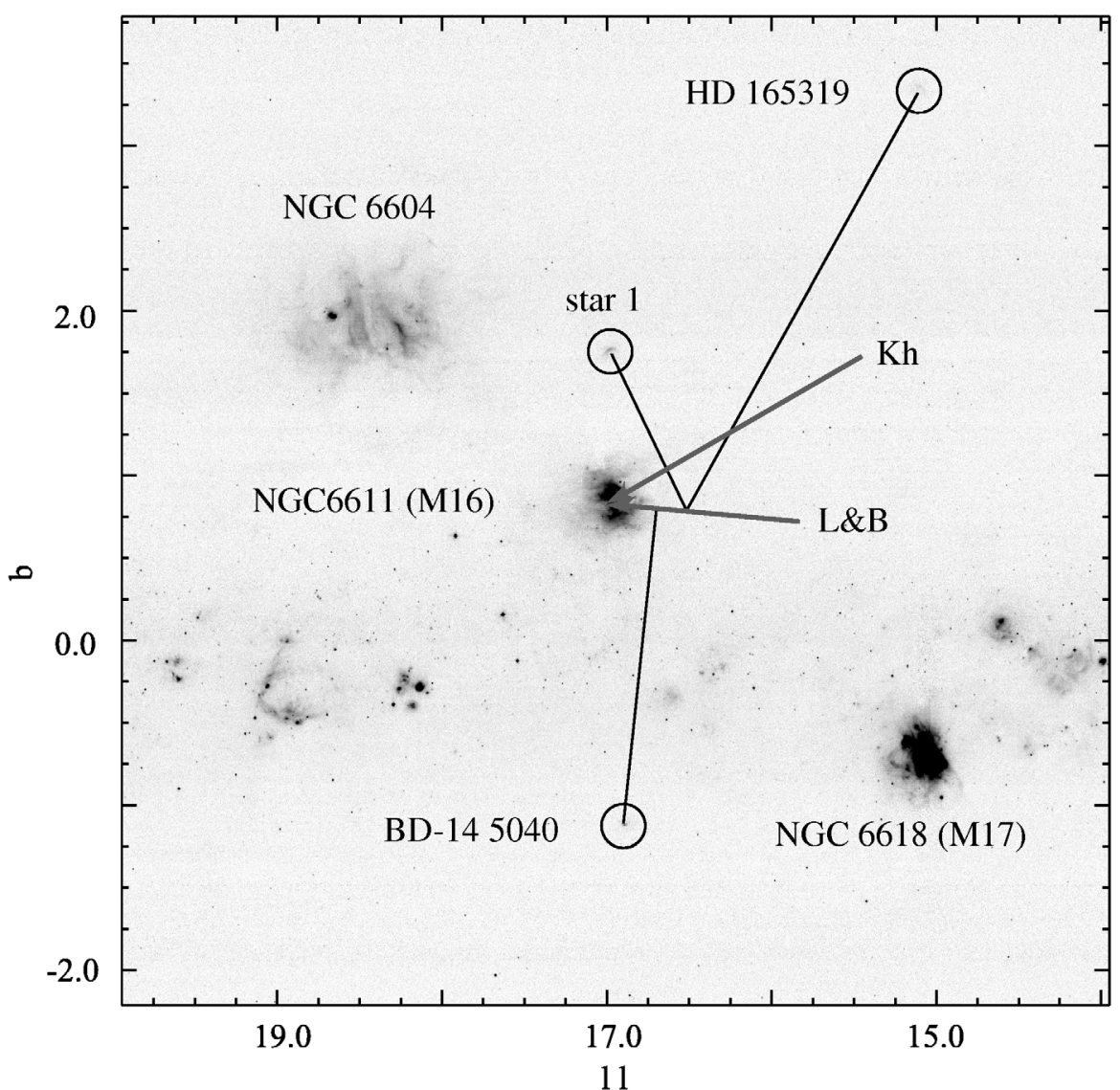

Fig. 1. A $6^{\circ} \times 6^{\circ} 21.3 \mu \mathrm{m}(M S X$ band E) image of NGC 6611 and its environments [including two open clusters NGC 6604 and NGC 6618 (M 17)], with the positions of three bow shocks marked by circles. The arrows labeled as L\&B and Kh show the direction of cluster's peculiar motion, based on the proper motion measurements, respectively, by Loktin \& Bechenov (2003) and Kharchenko et al. (2005). The origin of the arrows corresponds to possible birth places of the cluster $\sim 4$ Myr ago. The thin lines represent the trajectories of ejected stars, as suggested by the geometry of their bow shocks and proper motion measurements (see text for details). North is up and east to the left.

Table 1. Proper-motion measurements for NGC 6611 and the three ejected stars. Two measurements are given for each object to indicate the uncertainties in the measurements. For each data set, the peculiar (transverse) velocities (in Galactic coordinates) were calculated and added to the table.

\begin{tabular}{lccccc}
\hline \hline Object & $\begin{array}{c}\mu_{\alpha} \cos \delta \\
\mathrm{mas} \mathrm{yr}^{-1}\end{array}$ & $\begin{array}{c}\mu_{\delta} \\
\mathrm{mas} \mathrm{yr}^{-1}\end{array}$ & $\begin{array}{c}\text { Sources for } \\
\text { proper motions }\end{array}$ & $\begin{array}{c}v_{\mathrm{l}} \\
\mathrm{km} \mathrm{s}^{-1}\end{array}$ & $\begin{array}{c}v_{\mathrm{b}} \\
\mathrm{km} \mathrm{s}^{-1}\end{array}$ \\
\hline NGC 6611 & $0.6 \pm 0.1$ & $-0.3 \pm 0.1$ & Loktin \& Beshenov (2003) & $9.7 \pm 0.9$ & $0.7 \pm 0.9$ \\
NGC 6611 & $1.6 \pm 0.3$ & $-0.4 \pm 0.5$ & Kharchenko et al. (2005) & $12.9 \pm 4.3$ & $-7.7 \pm 3.5$ \\
star 1 & $0 \pm 7$ & $12 \pm 3$ & USNO-B1.0 (Monet et al. 2003) & $108.7 \pm 39.8$ & $61.5 \pm 59.7$ \\
star 1 & $-4.3 \pm 8.0$ & $-0.9 \pm 8.0$ & NOMAD (Zacharias et al. 2004a) & $-18.2 \pm 36.9$ & $39.2 \pm 36.9$ \\
BD-14 5040 & $5.5 \pm 1.3$ & $-3.0 \pm 1.4$ & NOMAD (Zacharias et al. 2004a) & $7.5 \pm 13.1$ & $-51.7 \pm 12.5$ \\
BD-14 5040 & $7.7 \pm 1.6$ & $-4.6 \pm 1.8$ & Kharchenko (2001) & $4.0 \pm 16.8$ & $-76.5 \pm 15.9$ \\
HD 165319 & $-1.2 \pm 1.0$ & $-1.4 \pm 0.9$ & UCAC2 (Zacharias et al. 2004b) & $-9.7 \pm 8.7$ & $11.0 \pm 9.3$ \\
HD 165319 & $0.3 \pm 1.0$ & $-1.8 \pm 0.6$ & NOMAD (Zacharias et al. 2004a) & $-6.1 \pm 6.7$ & $-3.3 \pm 8.7$ \\
\hline
\end{tabular}

\subsection{Associated stars}

The SIMBAD database provides the spectral types of two of the three stars associated with the bow shocks. We found that the bow shock 2 is driven by the star BD- $14^{\circ} 5040$ [with a photometrically estimated spectral type of O8V; Kilkenny (1993)] and the bow shock 3 is produced by the star HD 165319 (whose spectral type of O9.5Iab was derived spectroscopically by Crampton \& Fisher 1974). The bow shock 1 is apparently generated by a star with coordinates: $\alpha_{2000}=18^{\mathrm{h}} 15^{\mathrm{m}} 23^{\mathrm{s}} 97, \delta_{2000}=$ $-13^{\circ} 19^{\prime} 35^{\prime \prime} .8$ (hereafter, star 1; see Fig. 2). This star has the visual magnitude of 11.81 (McLean et al. 2000) and 2MASS magnitudes $\left(J, H, \mathrm{~K}_{\mathrm{s}}\right)=(8.096,7.684,7.396)($ Skrutskie et al. 2006). Using the extinction law from Rieke \& Lebofsky (1985) and the photometric calibration of optical and infrared magnitudes for Galactic O stars by Martins \& Plez (2006), we derived extinction towards the star 1 of $A_{\mathrm{V}} \simeq 5.6 \mathrm{mag}$ and estimated the spectral type of this star to be 09.5III/O5V (for $d=1.75 \mathrm{kpc}$ ) or $07.5 \mathrm{III} / \mathrm{O} 4 \mathrm{~V}(d=2.00 \mathrm{kpc})$. The spectral type of O7.5III is more consistent with the adopted age of the cluster of $4 \mathrm{Myr}$. Using the same calibration, we re-estimated the spectral type of BD-14 5040 to be $07 \mathrm{~V}(d=1.75 \mathrm{kpc})$ or O6V $(d=2.00 \mathrm{kpc})$ and derived extinction of $A_{\mathrm{V}} \simeq 3.8 \mathrm{mag}$. For both distances, the isochronal age of the star is less than $4 \mathrm{Myr}$, so that this star could be a blue straggler ejected from the cluster (e.g. Leonard 1995; Gvaramadze \& Bomans 2008). Also, we estimated the photometric distance to HD 165319 to be $d \simeq 2.00 \mathrm{kpc}$, in good agreement with the distance to NGC 6611 .

\subsection{Proper motions}

Figures 1, 2 and 4 show that the symmetry axes of the bow shocks 1 and 3 are misaligned with respect to the lines drawn through the associated stars and the center of the cluster. This 


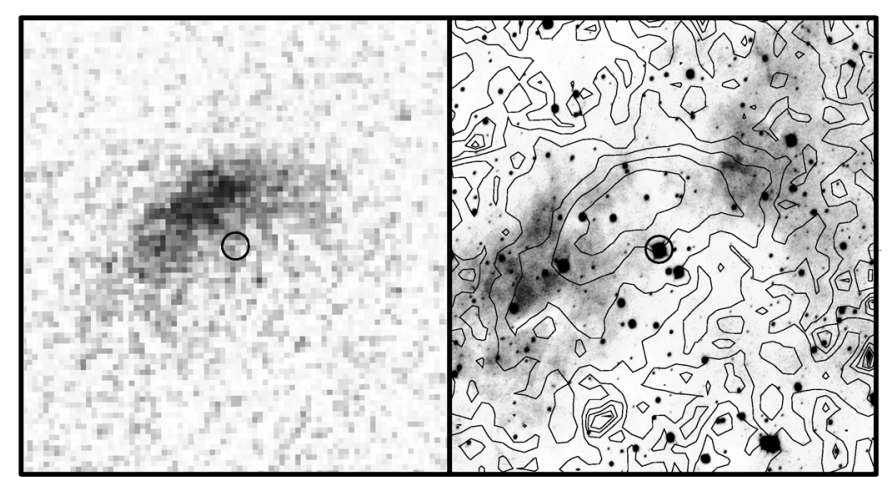

Fig. 2. Left: MSX $21.3 \mu \mathrm{m}$ image of the bow shock 1. The position of the associated star (star 1) is marked by a circle. Right: SHS image of the same field with the MSX $21.3 \mu \mathrm{m}$ image overlayed in black contours. Field size is $8^{\prime}$ by 8.5 , the images are oriented with Galactic 11 increasing to the left and Galactic b increasing upward.

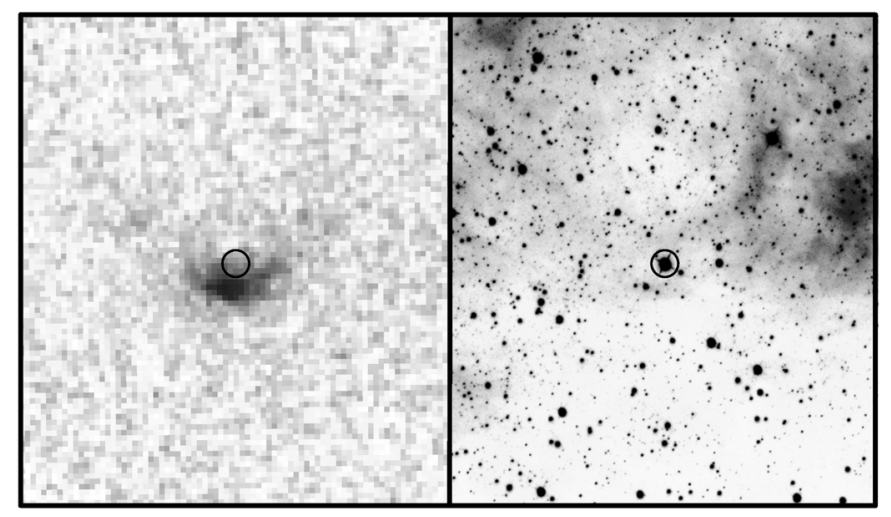

Fig. 3. Left: $M S X 21.3 \mu \mathrm{m}$ image of the bow shock 2. The position of the associated star BD $-14^{\circ} 5040$ is marked by a circle. Right: SHS image of the same field. Field size and orientation of this figure are the same as for Fig. 2.

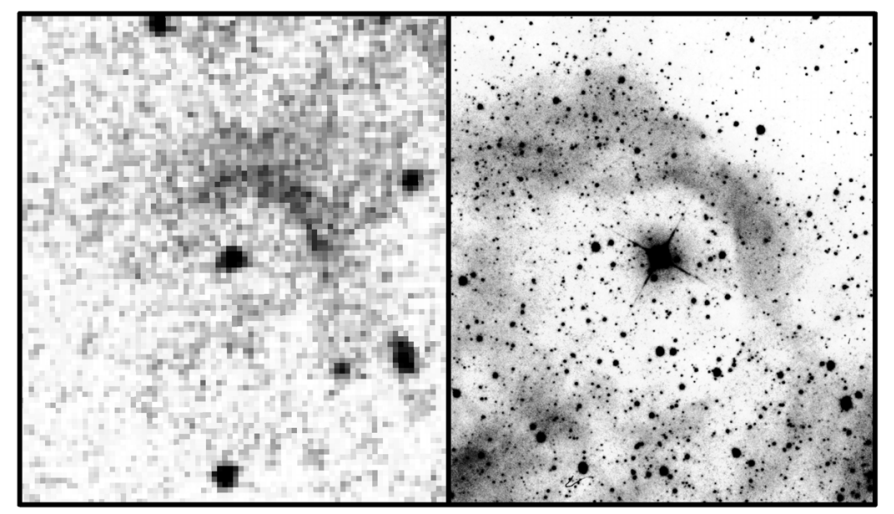

Fig. 4. Left: MSX $8.3 \mu \mathrm{m}$ image of the bow shock 3 and the associated star HD 165319. Right: SHS image of the same field. Field size and orientation of this figure are the same as for Fig 2.

misalignment could be understood if one takes into account the eastward motion of the cluster (see Sect. 2 and Fig. 1; cf. Hoogerwerf et al. 2001). Assuming that there is no bulk streaming motions of the ambient gas, we found that the trajectories of the star 1 and HD 165319 (suggested by the morphology of their bow shocks) intersect with the trajectory of the cluster $\simeq 1.6 \mathrm{Myr}$ ago, if one uses the cluster proper motion measurements by Loktin \& Bechenov (2003), or 0.9 Myr (star 1) and
1.8 Myr (HD 165319) ago for the proper motion measurements by Kharchenko et al. (2005). In both cases, the stars were ejected more than $2 \mathrm{Myr}$ after the cluster formation. This inference however would be incorrect if the ambient medium had a significant peculiar velocity, such that the symmetry axes of the bow shocks did not coincide with the direction of peculiar motion of the associated stars.

In Table 1, we provide two representative proper motion measurements for each star, found with the VizieR Catalogue Service. One can see that only BD- $14^{\circ} 5040$ has a significant proper motion (i.e. the measurement uncertainties are far less than the measurements themselves). Both measurements for this star suggest that it was ejected $\sim 1$ Myr ago with a velocity typical of classical runaway stars. It is curious that the symmetry axis of the bow shock produced by this star points almost exactly towards the cluster. If however the star was ejected from NGC 6611 and the proper motion measurements are correct, then the trajectory of the star should intersect with the cluster $\simeq 0.30^{\circ}-0.45^{\circ}$ to the west of its present position (for convenience, we show in Fig. 1 only one trajectory of the star). The "incorrect" orientation of the bow shock, in this case, could be attributed to the effect of peculiar motion of the ambient gas.

The proper motion measurements for the star 1 and HD 165319 are insignificant. Taken at face value, they suggest that the star 1 is a runaway in the classical sense and that it was able to reach its present position even if ejected from NGC 6611 only $\sim 0.9 \mathrm{Myr}$ ago (see above). The peculiar velocity of HD 165319 (see Table 1), however, is far smaller than the velocity ( of $\sim 45-60 \mathrm{~km} \mathrm{~s}^{-1}$ ) inferred from the separation of this star from the possible trajectories of the cluster and the ejection time implied by the morphology of its bow shock. This situation cannot be "improved" if one takes into account that the true (ejection) velocity of HD 165319 (i.e. the peculiar velocity in the reference frame of the cluster) is higher than the "measured" velocity (given in the last two columns of Table 1). Since the available proper motion measurements for this star are highly unreliable and the symmetry axis of its bow shock could deviate from the direction of stellar peculiar motion, one cannot however exclude that either the true peculiar velocity of HD 165319 is much higher than the "measured" one or that this star was ejected soon after the cluster formation.

\section{Discussion}

Our search for OB stars running away from the young open cluster NGC 6611 has resulted in the discovery of three bow shocks generated by massive stars. The morphology of the bow shocks suggest that the associated stars were ejected from NGC 6611. All three stars are of O-type, which is consistent with the observational fact that the percentage of $\mathrm{O}$ stars among runaways is higher than that of B stars (e.g. Stone 1991).

It is natural to assume that the three stars were ejected when the central density in NGC 6611 was sufficiently high for close dynamical encounters between the cluster members to be frequent. Whether or not the high star density in the cores of young clusters is primordial (i.e. intrinsic to the cluster formation process, e.g. Murray \& Lin 1996; Clarke \& Bonnell 2008) or is the result of the mass-segregation instability (e.g. Portegies Zwart et al. 1999, Gürkan et al. 2004) remains unclear to date (e.g. Kroupa 2008). In the first case, the cluster could be collisional at birth, so that one can expect that the majority of runaway stars were produced at the very beginning of cluster evolution. In this case, the kinematic age of ejected stars should be comparable with the age of the cluster. In the second case, the beginning of 
the collisional stage is delayed for several Myr and the kinematic age of runaway stars is younger than the age of the parent cluster. Accurate proper motion measurements of runaway stars and their parent clusters are needed to distinguish between these two possibilities.

The large separation between HD 165319 and NGC 6611 and the low peculiar velocity of the star might imply that it was ejected soon after the cluster formation, supporting the first possibility. The proper motion measurements for this star are however unreliable (see Table 1), which leaves a possibility that HD 165319 was ejected recently, as suggested by the symmetry axis of its bow shock. The late production of runaway stars also follows from the young kinematic age of BD-14 5040 (the only one of the three stars with reliable proper motion measurements) and might be inferred from the morphology of the bow shock produced by star 1 . The available astrometric data, however, do not allow us to differentiate between whether or not NGC 6611 experienced a burst of star ejection $\sim 1-2$ Myr ago or this process continued up until cluster formation. Indirect support of the latter possibility comes from the study of field $\mathrm{O}$ stars by Schilbach $\&$ Röser (2008). These authors retraced the orbit of the O-type star HD 157857 in the Galactic potential and found that this star was ejected from NGC $6611 \simeq 3.8 \mathrm{Myr}$ ago with a peculiar velocity of $\simeq 114 \mathrm{~km} \mathrm{~s}^{-1}$. More robust proper motion measurements for star 1 and HD 165319 would help to solve the problem.

By assuming that all three stars were ejected from NGC 6611 and taking into account that $\$ 20$ per cent of runaway stars produce bow shocks (see Sect. 1), one can infer that at least 15 massive stars have been lost by the cluster during its lifetime. The true number of ejected $\mathrm{OB}$ stars could be far higher since high-velocity runaways would have already traveled beyond the region covered by the $M S X$ survey $^{1}$, while the more numerous stars ejected at low velocities $\left(\lesssim 30 \mathrm{~km} \mathrm{~s}^{-1}\right.$ ) have lower probabilities of their motion relative to the ambient medium being supersonic and therefore of being able to generate the bow shocks. We therefore believe that the percentage of stars producing bow shocks could be far lower than 20 per cent, based on the study of "classical" runaway stars. From this follows that the number of ejected OB stars could be comparable with or higher than the number still residing in NGC 6611, which could explain the apparent deficit of OB stars in the cluster (see Sect. 2).

In addition to the dynamical ejection, there are two other effects that can reduce the size of the $\mathrm{OB}$ star population in the cluster: (i) supernova explosions of the most massive stars and (ii) merging of stars in the course of close binary-single and binary-binary star encounters. Supernova explosions in massive binaries could be accompanied by ejection of companion stars (e.g. Blaauw 1961; Leonard \& Dewey 1993), which in addition reduce the population of massive stars in the cluster. Supernova explosions begin to occur in clusters of age $\gtrsim 3$ Myr. Flagey (2007) revealed a shell-like structure within the Eagle Nebula and suggested that this shell was a young ( 3000 yr) supernova remnant. If confirmed, this suggestion would imply that NGC 6611 is at least as old as 3 Myr, which is consistent with the age of the cluster adopted in Sect. 2. For the age and mass of NGC 6611 of $\simeq 4 \mathrm{Myr}$ and $\simeq 2 \times 10^{4} M_{\odot}$, respectively, and assuming a Salpeter initial mass function, one finds that $\simeq 10$ stars have exploded as supernovae since the cluster formation. Correspondingly, the number of massive runaways produced by binary disruptions following supernova explosions cannot exceed this number. Merging of stars is a by-product of star

\footnotetext{
1 A possible example of such a high-velocity runaway is the aforementioned star HD 157857, located at $\sim 13^{\circ}$ from NGC 6611 .
}

ejection by means of binary-single and binary-binary encounters (e.g. Leonard 1995). Four early-type O stars in NGC 6611 and the star producing the bow shock 2 (BD-14 $\left.{ }^{\circ} 5040\right)$ appear younger than the main population of $\mathrm{OB}$ stars in the cluster and therefore could be the rejuvenated merged stars (e.g. Leonard 1995; Portegies Zwart et al. 1999; Gvaramadze \& Bomans 2008). Therefore, we suggest that at least 5 OB stars were "lost" by the cluster via merging.

We note that HD 165319 was considered by de Wit et al. (2005) to be one of "the best examples for isolated Galactic highmass star formation". de Wit et al. $(2004,2005)$ searched for parent star clusters for 43 O-type field stars to confirm whether or not these massive stars originated in a clustered mode of star formation. They found that 11 stars from their sample cannot be associated with a nearby cluster or star-forming region and suggested that $\sim 4 \pm 2$ per cent of all field O-type stars could be formed in isolation (cf. Chu \& Gruendl 2008). If confirmed, this result could have important implications for understanding massive star formation. It would either imply the existence of a special mode of isolated star formation (e.g. Yorke \& Sonnhalter 2002; Li et al. 2003) or allow the possibility that low mass $\left(<100 M_{\odot}\right)$ clusters are able to form O stars (e.g. Elmegreen 2006; Parker \& Goodwin 2007).

de Wit et al. (2005) restricted the search for parent clusters within a circle of radius $65 \mathrm{pc}$; this figure comes from the average peculiar radial velocity of field $\mathrm{O}$ stars, which is $6.4 \mathrm{~km} \mathrm{~s}^{-1}$ (Gies 1987), multiplied by their average lifetime of $10 \operatorname{Myr}\left(1 \mathrm{~km} \mathrm{~s}^{-1} \times 1 \mathrm{Myr} \simeq 1 \mathrm{pc}\right)$. It is possible therefore that at least some of the 11 stars, suggested by de Wit et al. (2005) to be formed in isolation, were instead ejected from clusters more distant than $65 \mathrm{pc}$. The discovery of the bow shock around HD 165319 and identification of its possible parent cluster (separated by $\simeq 105 \mathrm{pc}$ ) lends support to this suggestion. We note that some of the remaining 10 stars could be rejuvenated stars (blue stragglers) formed via merging of less massive stars in binaries ejected from clusters located far beyond the circle of radius of 65 pc. Study of field O-type stars by Schilbach \& Röser (2008) suggests that 6 of these 10 stars were ejected from open star clusters and were therefore formed in the clustered way. In some cases, however, the times-of-flight derived by Schilbach \& Röser (2008) exceed the lifetimes of the stars. The proposed identification of the parent clusters would therefore be incorrect if these stars are not blue stragglers.

We searched for bow shocks around the remaining 10 O-type stars formed in "isolation" using the $M S X$ survey but none were found (note that the survey covers only 8 of 10 stars). This nondetection could be due to the limited and inhomogeneous sensitivity of the survey (see Price et al. 2001). It is also possible that some of these stars have not produced bow shocks simply because their peculiar velocities are less than the sound speed in the (hot) ambient interstellar medium (see Sect. 1). We note that the peculiar velocities relative to the ambient medium (and therefore ability to generate bow shocks) of some runaway stars could be affected by the cluster peculiar motion. A (massive) star ejected in the direction opposite to the cluster's motion will have a reduced peculiar velocity relative to the local standard of rest, while its true (ejection) velocity with respect to the cluster could be sufficiently high to classify the star as a "classical" runaway. This star has not only a lower probability of producing a bow shock, but is also more distant from the parent cluster than one can infer from its apparent proper motion. Since most ejected stars have velocities of several tens $\mathrm{km} \mathrm{s}^{-1}$ (i.e. comparable with the escape velocity from the parent cluster) and the peculiar velocities of clusters could be as high as $\sim 10-20 \mathrm{~km} \mathrm{~s}^{-1}$ 


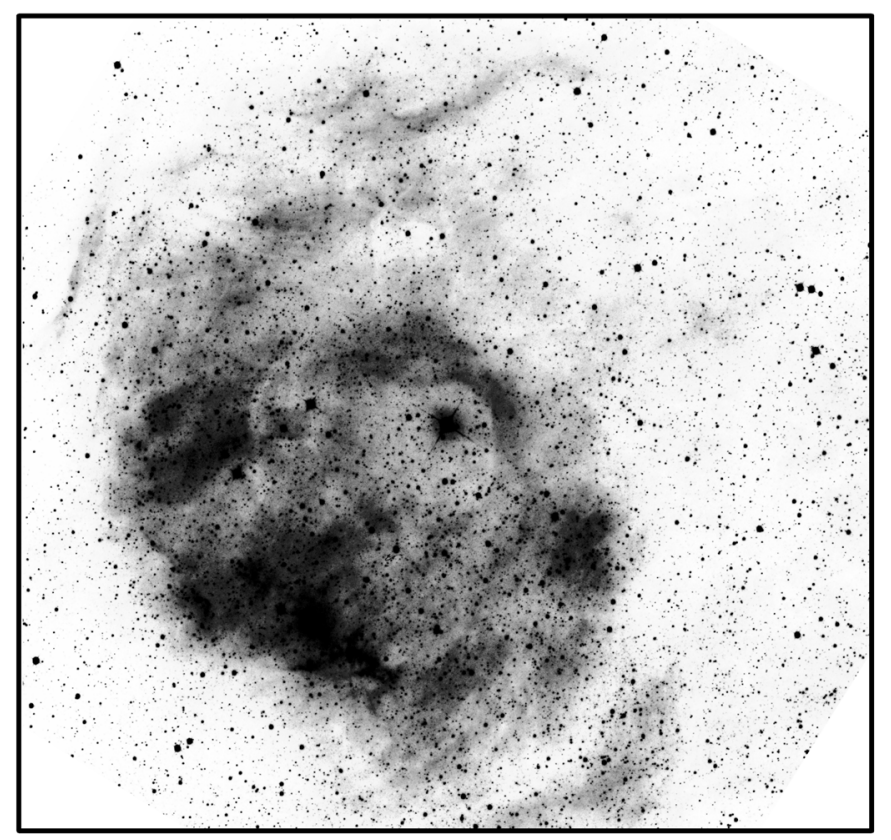

Fig. 5. A $0.5 \times 0.5$ SHS image of RCW 158, again oriented in Galactic coordinates (increasing 11 to left, and increasing b upward).

(de Zeeuw et al. 1999; see also Sect. 2), the above effect could be significant and might cause an excess of bow-shock-producing stars ahead of the moving cluster.

In Sect. 3.1, we mentioned that the bow shock 3 is part of the $\mathrm{H}$ II region RCW158. de Wit et al. (2005) consider this nebula as the tracer of isolated high mass star formation. The almost central location of HD 165319 in RCW 158 (Fig. 5) suggests, however, that RCW 158 might be a Strömgren zone created by the ultraviolet emission of the star. We now check this possibility. Assuming that the gas in RCW 158 is fully ionized and its temperature is $10^{4} \mathrm{~K}$, one can obtain an estimate of the gas number density (e.g. Lequeux 2005)

$n \simeq 22 \mathrm{~cm}^{-3}\left(\frac{R_{\mathrm{S}}}{6.88 \mathrm{pc}}\right)^{-3 / 2}\left(\frac{S(0)}{4.90 \times 10^{48} \text { photons s}^{-1}}\right)^{1 / 2}$,

where $R_{\mathrm{S}}$ is the Strömgren radius (derived from the angular radius of RCW 158 of $\sim 12^{\prime}$ and $d=2 \mathrm{kpc}$ ) and $S(0)$ is the total ionizing-photon luminosity of an O9.5Iab star (taken from Martins et al. 2005). This value should be equal to that derived from the ram pressure balance between the stellar wind and the ambient medium:

$$
\begin{array}{r}
n \simeq 17 \mathrm{~cm}^{-3}\left(\frac{\dot{M}}{5.3 \times 10^{-6} M_{\odot} \mathrm{yr}^{-1}}\right)\left(\frac{v_{\infty}}{2100 \mathrm{~km} \mathrm{~s}^{-1}}\right) \\
\times\left(\frac{R_{0}}{0.8 \mathrm{pc}}\right)^{-2}\left(\frac{v_{*}}{50 \mathrm{~km} \mathrm{~s}^{-1}}\right)^{-2},
\end{array}
$$

where $\dot{M}$ and $v_{\infty}$ are the wind mass-loss rate and terminal velocity of an O9.5Iab star (taken from Mokiem et al. 2007), $R_{0}$ is the stand-off radius ${ }^{2}$, and $v_{*}$ is the velocity of the star relative to the ambient medium, assumed to be equal to $50 \mathrm{~km} \mathrm{~s}^{-1}$ (see Sect. 3.3). The good agreement between the two estimates supports our suggestion that RCW 158 is the Strömgren zone of

\footnotetext{
${ }^{2}$ We neglected here a geometric factor of order unity, taking into account the inclination of the bow shock due to the peculiar radial velocity of the star of $\simeq 20.5 \mathrm{~km} \mathrm{~s}^{-1}$ (derived from the measured radial velocity of $\simeq 25.4 \mathrm{~km} \mathrm{~s}^{-1}$ (Kharchenko et al. 2007)).
}

HD 165319. The stellar parameters used in Eq. (2) could however still vary within the classification [see e.g. the values given by Markova et al. (2004) for the same spectral and luminosity class]. Using different possible values for the stellar parameters and allowing the velocity to be between $30 \mathrm{~km} \mathrm{~s}^{-1}$ and $60 \mathrm{~km} \mathrm{~s}^{-1}$ in Eq. (2), we estimate the approximate lower and upper bounds of the density to be $10 \mathrm{~cm}^{-3}$ and $30 \mathrm{~cm}^{-3}$, respectively. This density range is again in good agreement with the density estimate from the Strömgren formula (Eq. (1)). Further support comes from the observation that RCW 158 is partially surrounded by $8.3 \mu \mathrm{m}$ filamentary structures, which are typical of photodissociation regions (Hollenbach \& Tielens 1997). We therefore conclude that the ionizing photons emitted by HD 165319 have created a Strömgren sphere in the dense ambient medium (visible as RCW 158), while the interaction between the wind of this supersonically moving star and the ambient gas generates a bow shock ahead of the star and evacuates a cavity behind (see Fig. 5; cf. Raga et al. 1997).

\section{Conclusions}

With the detection of three bow-shock-producing stars in the vicinity of NGC 6611, we have found strong evidence that the proposed loss of massive stars, due to dynamical processes in the early evolution of young clusters, is indeed operating. While existing astrometric data for the three stars do not allow us to determine precisely the timing of the ejection or study the interaction of the runaway stars with the ISM in detail, the morphology of the bow shocks and the proper motion of the cluster itself allows us to argue that these stars originated in NGC 6611.

Clearly, our finding opens up a number of possibilities for follow-up investigations of the NGC 6611 bow shocks and the ejected stars. More accurate proper motion measurements will allow us to check if the stars were ejected continuously or during a short period of cluster evolution. The most important point nevertheless is that the idea proposed by Pflamm-Altenburg \& Kroupa (2006) is supported by direct observation, at least in the case of NGC 6611. The results for several other young clusters will be presented in forthcoming papers.

Acknowledgements. The authors are grateful to P. Kroupa, F. Martins and J Pflamm-Altenburg for interesting discussions, to the anonymous referee for suggestions allowing us to improve the manuscript and to N. Flagey for sending us his Ph.D. Thesis. This research has made use of the NASA/IPAC Infrared Science Archive, which is operated by the Jet Propulsion Laboratory, California Institute of Technology, under contract with the National Aeronautics and Space Administration, the SIMBAD database and the VizieR catalogue access tool, both operated at CDS, Strasbourg, France. The authors acknowledge financial support from the Deutsche Forschungsgemeinschaft (grants 436 RUS 17/104/06 and BO 1642/14-1) for research visits of V.V.G. at the Astronomical Institute of the Ruhr-University Bochum.

\section{References}

Aarseth, S. J., \& Hills, J. G. 1972, A\&A, 21, 255

Avedisova, V. S. 2005, Astron. Rep., 49, 435

Ballesteros-Paredes, J., \& Hartmann, L. 2007, Rev. Mex. Astron. Astrofis., 43, 123

Baranov, V. B., Krasnobaev, K. V., \& Kulikovskii, A. G. 1971, Sov. Phys. Dokl., 15,791

Belikov, A. N., Kharchenko, N. V., Piskunov, A. E., \& Schilbach, E. 2000, A\&A, 358,886

Blaauw, A. 1961, Bull. Astron. Inst. Netherl., 15, 265

Boily, C. M., \& Kroupa, P. 2003, MNRAS, 338, 665

Bonatto, C., Santos, Jr., J. F. C., \& Bica, E. 2006, A\&A, 445, 567

Bosch, G. L., Morrell, N. I., \& Niemelä, V. S. 1999, Rev. Mex. Astron. Astrofis., 35,85

Brown, D., \& Bomans, D. J. 2005, A\&A, 439, 183 
Chen, L., de Grijs, R., \& Zhao, J. L. 2007, AJ, 134, 1368

Chu, Y.-H., \& Gruendl, R. A. 2008, in Massive Star Formation: Observations Confront Theory, eds. H. Beuther, H. Linz, \& T. Henning, ASP Conf. Ser., 387,415

Clarke, C. J., \& Bonnell, I. A. 2008, MNRAS, 388, 1171

Crampton, D., \& Fisher, W. A. 1974, Publ. Dom. Astrophys. Obs., 14, 283

de Winter, D., Koulis, C., Thé, P. S., et al. 1997, A\&AS, 121, 223

de Wit, W. J., Testi, L., Palla, F., Vanzi, L., \& Zinnecker, H. 2004, A\&A, 425, 937

de Wit, W. J., Testi, L., Palla, F., \& Zinnecker, H. 2005, A\&A, 437, 247

de Zeeuw, P. T., Hoogerwerf, R., de Bruijne, J. H. J., Brown, A. G. A., \& Blaauw, A. 1999, AJ, 117, 354

Dehnen, W., \& Binney, J. J. 1998, MNRAS, 298, 387

Dufton, P. L., Smartt, S. J., Lee, J. K., et al. 2006, A\&A, 457, 265

Edelmann, H., Napiwotzki, R., Heber, U., Christlieb, N., \& Reimers, D. 2005, ApJ, 634, L181

Elmegreen, B. G. 2000, ApJ, 530, 277

Elmegreen, B. G. 2006, ApJ, 648, 572

Flagey, N. 2007, Ph.D. Thesis, Université Paris Sud

France, K., McCandliss, S. R., \& Lupu, R. E. 2007, ApJ, 655, 920

Gies, D. R. 1987, ApJS, 64, 545

Gouliermis, D., Keller, S. C., Kontizas, M., Kontizas, E., \& Bellas-Velidis, I. 2004, A\&A, 416, 137

Guarcello, M. G., Prisinzano, L., Micela, G., et al. 2007, A\&A, 462, 245

Gürkan, M. A., Freitag, M., \& Rasio, F. A. 2004, ApJ, 604, 632

Gvaramadze, V. V., \& Bomans, D. J. 2008, A\&A, 485, L29

Heber, U., Edelmann, H., Napiwotzki, R., Altmann, M., \& Scholz, R.-D. 2008, A\&A, 483, L21

Hester, J. J., Gilmozzi, R., O’Dell, C. R., et al. 1996, ApJ, 369, L75

Hillenbrand, L. A., \& Hartmann, L. W. 1998, ApJ, 492, 540

Hillenbrand, L., Massey, P., Strom, S. E., \& Merrill, K. M. 1993, AJ, 106, 1906

Hollenbach, D. J., \& Tielens, A. G. G. M. 1997, ARA\&A, 35, 179

Hoogerwerf, R., de Bruijne, J. H. J., \& Zeeuw, P. T. 2001, A\&A, 365, 49

Huthoff, F., \& Kaper, L. 2002, A\&A, 383, 999

Kalirai, J. S., Richer, H. B., Hansen, B. M., et al. 2004, ApJ, 601, 277

Kaper, L., van Loon, J.Th., Augusteijn, T., et al. 1997, ApJ, 475, L37

Kharchenko, N. V. 2001, Kinematika Fiz. Nebesnykh Tel, 17, 409

Kharchenko, N. V., Piskunov, A. E., Röser, S., Schilbach, E., \& Scholz, R.-D. 2005, A\&A, 438, 1163

Kharchenko, N. V., Scholz, R.-D., Piskunov, A. E., Röser, S., \& Schilbach, E. 2007, AN, 328, 889

Kilkenny, D. 1993, S. Afr. Astron. Obs. Circ., 15, 53

Kroupa, P. 2004, New Astron. Rev., 48, 47

Kroupa, P. 2008, preprint [arXiv:0803.1833]

Kroupa, P., \& Boily, C. M. 2002, MNRAS, 336, 1188
Kuchar, T. A., \& Clark, F. O. 1997, ApJ, 488, 224

Lequeux, J. 2005, The Interstellar Medium (Berlin: Springer)

Leonard, P. J. T. 1991, AJ, 101, 562

Leonard, P. J. T. 1995, MNRAS, 277, 1080

Leonard, P. J. T., \& Dewey, R. J. 1993, in Luminous High-Latitude Stars, ed. D. Sasselov, ASP Conf. Ser., 45, 239

Leonard, P. J. T., \& Duncan, M. J. 1990, AJ, 99, 608

Li, Y., Klessen, R. S., \& Mac Low, M.-M. 2003, ApJ, 592, 975

Loktin, A. V., \& Beshenov, G. V. 2003, Astron. Rep., 47, 6

Markova, N., Puls, J., Repolust, T., \& Markov, H. 2004, A\&A, 413, 693

Martins, F., \& Plez, B. 2006, A\&A, 457, 637

Martins, F., Schaerer, D., \& Hillier, D. J. 2005, A\&A, 436, 1049

McLean, B. J., Greene, G. R., Lattanzi, M. G., \& Pirenne, B. 2000, in Astronomical Data Analysis Software and Systems IX, eds. N. Manset, C. Veillet, \& D. Crabtree, ASP Conf. Ser., 216, 145

Mikkola, S. 1983, MNRAS, 205, 733

Mokiem, M. R., de Koter, A., Vink, J. S., et al. 2007, A\&A, 473, 603

Monet D. G., Levine S. E., Casian B., et al. 2003, AJ, 125, 984

Murray, S. D., \& Lin, D. N. C. 1996, ApJ, 467, 728

Noriega-Crespo, A., Van Buren, D., \& Dgani, R. 1997, AJ, 113, 780

Parker, Q. A., Phillipps, S., Pierce, M. J., et al. 2005, MNRAS, 362, 689

Parker, R. J., \& Goodwin, S. P. 2007, MNRAS, 380, 1271

Portegies Zwart, S. F., Makino, J., McMillan, S. L. W., \& Hut, P. 1999, A\&A, 348, 117

Poveda, A., Ruiz, J., \& Allen, C. 1967, Bol. Obs. Tonantzintla Tacubaya, 4, 86 Pflamm-Altenburg, J., \& Kroupa, P. 2006, MNRAS, 373, 295

Pflamm-Altenburg, J., \& Kroupa, P. 2007, MNRAS, 375, 855

Price, S. D., Egan, M. P., Carey, S. J., Mizuno, D. R., \& Kuchar, T. A. 2001, AJ, 121,2819

Raga, A. C., Noriega-Crespo, A., Cantó, J., et al. 1997, Rev. Mex. Astron. Astrofis., 33, 73

Ramspeck, M., Heber, U., \& Moehler, S. 2001, A\&A, 378, 907

Reid, M. J. 1993, ARA\&A, 31, 345

Rieke, G. H., \& Lebofsky, M. J. 1985, ApJ, 288, 618

Rodgers, A. W., Campbell, C. T., \& Whiteoak, J. B. 1960, MNRAS, 121, 103

Schilbach, E., \& Röser, S. 2008, A\&A, 489, 105

Skrutskie, M. F., Cutri, R. M., Stiening, R., et al. 2006, AJ, 131, 1163

Stone, R. C. 1991, AJ, 102, 333

Van Buren, D., \& McCray, R. 1988, ApJ, 329, L93

Van Buren, D., Noriega-Crespo, A., \& Dgani, R. 1995, AJ, 110, 2914

Wolff, S. C., Strom, S. E., Dror, D., \& Venn, K. 2007, AJ, 133, 1092

Yorke, H. W., \& Sonnhalter, C. 2002, ApJ, 569, 846

Zacharias, N., Monet, D. G., Levine, S. E., et al. 2004a, A\&AS, 205, 4815

Zacharias, N., Urban, S. E., Zacharias, M. I., et al. 2004b, AJ, 127, 3043 